OPEN ACCESS

Edited by:

Daniela Ceccarelli, Research Executive Agency,

European Commission, Belgium

Reviewed by:

Andres Felipe Opazo-Capurro, Universidad de Concepción, Chile Carlos Henrique Camargo,

Instituto Adolfo Lutz, Brazil

*Correspondence: Ghassan M. Matar gmatar@aub.edu.lb

Specialty section: This article was submitted to Antimicrobials, Resistance and Chemotherapy, a section of the journal Frontiers in Microbiology

Received: 11 February 2019 Accepted: 12 April 2019

Published: 30 April 2019

Citation:

El Hafi B, Rasheed SS, Abou Fayad AG, Araj GF and Matar GM (2019) Evaluating the Efficacies

of Carbapenem/ $\beta$-Lactamase Inhibitors Against

Carbapenem-Resistant

Gram-Negative Bacteria in vitro and in vivo. Front. Microbiol. 10:933.

doi: 10.3389/fmicb.2019.00933

\section{Evaluating the Efficacies of Carbapenem/ $\beta$-Lactamase Inhibitors Against Carbapenem-Resistant Gram-Negative Bacteria in vitro and in vivo}

\author{
Bassam El Hafi 1,2, Sari S. Rasheed ${ }^{1,2}$, Antoine G. Abou Fayad ${ }^{1,2}$, George F. Araj ${ }^{2,3}$ and \\ Ghassan M. Matar ${ }^{1,2 *}$ \\ ' Department of Experimental Pathology, Immunology and Microbiology, American University of Beirut, Beirut, Lebanon, \\ ${ }^{2}$ Center for Infectious Diseases Research, American University of Beirut, Beirut, Lebanon, ${ }^{3}$ Department of Pathology \\ and Laboratory Medicine, American University of Beirut Medical Center, Beirut, Lebanon
}

Background: Carbapenem-resistant Gram-negative bacteria are a major clinical concern as they cause virtually untreatable infections since carbapenems are among the last-resort antimicrobial agents. $\beta$-Lactamases implicated in carbapenem resistance include KPC, NDM, and OXA-type carbapenemases. Antimicrobial combination therapy is the current treatment approach against carbapenem resistance in order to limit the excessive use of colistin; however, its advantages over monotherapy remain debatable. An alternative treatment strategy would be the use of carbapenem/ $\beta$-lactamase inhibitor $(\beta L \mathrm{I})$ combinations. In this study, we assessed the in vitro and in vivo phenotypic and molecular efficacies of three $\beta$ LIs when combined with different carbapenems against carbapenem-resistant Gram-negative clinical isolates. The chosen $\beta$ LIs were (1) Avibactam, against OXA-type carbapenemases, (2) calcium-EDTA, against NDM-1, and (3) Relebactam, against KPC-2.

Methods: Six Acinetobacter baumannii clinical isolates were screened for blaOXA-23-like, blaOXA-24/40, blaOXA-51-like, blaOXA-58, and blaOXA-143-like, and eight Enterobacteriaceae clinical isolates were screened for blaOXA-48, bla NDM-1, and blakPC-2. The minimal inhibitory concentrations of Imipenem (IPM), Ertapenem (ETP), and Meropenem (MEM) with corresponding $\beta$ LIs for each isolate were determined. The efficacy of the most suitable in vitro treatment option against each of blaOXA-48, bla ${ }_{N D M-1}$, and bla K $_{K C-2}$ was assessed via survival studies in a BALB/c murine infection model. Finally, RT-qPCR was performed to assess the molecular response of the genes of resistance to the carbapenem/ $\beta \mathrm{LI}$ combinations used under both in vitro and in vivo settings.

Results: Combining MEM, IPM, and ETP with the corresponding $\beta$ LIs restored the isolates' susceptibilities to those antimicrobial agents in $66.7 \%, 57.1 \%$, and $30.8 \%$ of the samples, respectively. Survival studies in mice revealed $100 \%$ survival rates when MEM was combined with either Avibactam or Relebactam against blaoxA-48 
and blakPC-2, respectively. RT-qPCR demonstrated the consistent overexpression of bla $O X A-48$ upon treatment, without hindering Avibactam's activity, while bla $a_{N D M-1}$ and $b_{1} a_{K P C}-2$ experienced variable expression levels upon treatment under in vitro and in vivo settings despite their effective phenotypic results.

Conclusion: New carbapenem/ $\beta \mathrm{LI}$ combinations may be viable alternatives to antimicrobial combination therapy as they displayed high efficacy in vitro and in vivo. Meropenem/Avibactam and Meropenem/Relebactam should be tested on larger sample sizes with different carbapenemases before progressing further in its preclinical development.

Keywords: OXA-48, NDM-1, KPC, carbapenem, Avibactam, Relebactam, calcium-EDTA, antimicrobial resistance

\section{INTRODUCTION}

Carbapenem resistant Gram-negative bacteria have been gradually increasing in prevalence in recent years. In the United States, the latest CDC Antibiotic Resistance Threat Report indicates that Carbapenem-Resistant Enterobacteriaceae (CREs) are responsible for 9,000 annual nosocomial infections, with a $6.67 \%$ mortality rate; a potentially underestimated percentage due to different definitions of CRE infections (Livorsi et al., 2018). The same report also estimates 7,300 annual multidrug-resistant (MDR) Acinetobacter baumannii infections; with a $6.85 \%$ mortality rate. In Lebanon, the most recent nation-wide survey indicates that around 2\% of Enterobacteriaceae isolates identified over the past few years were Imipenem-resistant, while that percentage was much higher among Acinetobacter spp. at $82.4 \%$ (Chamoun et al., 2016). At the American University of Beirut Medical Center (AUBMC), the prevalence of CREs has doubled since 2015, reaching $11 \%$, while carbapenem resistance among A. baumannii isolates has remained high beyond $75 \%$ during the same time period (Araj and Zaatari, 2015, 2018).

Carbapenem resistance can manifest through several mechanisms. Notably, the combined effect of extended-spectrum $\beta$-lactamases (ESBLs) or AmpC-type enzymes production, coupled with increased efflux pump activity and porin loss (Baroud et al., 2013). However, the main mechanism of resistance to carbapenems is through the expression of chromosomal or plasmid-mediated carbapenem-hydrolyzing $\beta$-lactamases such as Klebsiella pneumoniae carbapenemases (KPC), OXA-type carbapenemases, and New Delhi metallo- $\beta$-lactamases (Lapuebla et al., 2015) (Meletis, 2016). KPC and OXA-type carbapenemases are families of Ambler Class A and Class D serine $\beta$-lactamases, respectively, that contain a serine moiety in their active sites (Sahuquillo-Arce et al., 2015). Among the KPC family, KPC-2 and KPC-3 are the most commonly encountered between the 20-plus variant KPCs (Djahmi et al., 2014; Sahuquillo-Arce et al., 2015; Satlin et al., 2017). The OXA-type carbapenemases are grouped into nine clusters with $1,2,3$, and 4 being associated with A. baumannii, and include the subfamilies OXA-23, OXA-51, OXA-24/40, and OXA-58, while cluster 6, being associated with Enterobacteriaceae, comprises the subfamily OXA-48 (Woodford et al., 2006; Queenan and Bush, 2007). On the other hand, NDM is a family of Ambler Class B metallo- $\beta$-lactamases that contain a divalent cation in their active site (Sahuquillo-Arce et al., 2015) with NDM-1 being the most prominent member (Nordmann et al., 2011).

Treating carbapenem-resistant Gram-negative bacteria poses a major clinical challenge as carbapenems are among the last-resort antimicrobial agents to be used, and CREs along with MDR-A. baumannii can cause terminal infections ranging from upper and lower respiratory, wound, bloodstream and cerebrospinal fluid infections in the case of A. baumannii (Queenan et al., 2012), to complicated intra-abdominal infections, sepsis, and meningitis, in the case of CREs (Murray et al., 2016; Yu and Chuang, 2016). The current recommendation to treat carbapenem-resistant Gram-negative infections involves the use of antimicrobial combination therapy (The Medical Letter, 2013). This approach is mostly guided by the lack of new classes of antimicrobial agents that can overcome such resistance since it is usually compounded with fluoroquinolone as well as aminoglycoside resistances within the same isolate (Meletis, 2016). Consequently, nephrotoxic antimicrobial agents such as polymyxins have to be combined with tetracyclines, such as tigecycline (Meletis, 2016). However, the efficacy of antimicrobial combination therapy in comparison to monotherapy has been a topic of debate in the literature. One study concluded that combination therapy improved the survival rates of bloodstream infection patients and decreased their mortality rates by $20.2 \%$ $(p=0.02)$ when compared to monotherapy (Tumbarello et al., 2012). Another study conducted on 205 patients infected with KPC-producing K. pneumoniae determined that combination therapy decreased patient mortality rate from 40 to $19.4 \%$ when a carbapenem is used in addition to other antimicrobials (Daikos et al., 2014). However, there exists sources of bias in combination therapy reports since a lot of studies include both carbapenem-resistant and carbapenem-susceptible isolates, not to mention that they disregard empirical treatment that the patient might have taken prior to being enrolled in the study (Paul et al., 2014). Additionally, certain studies report that the use of carbapenems as part of a double or triple therapy is recommended when the MIC needed against the isolate is $\leq 8$ $\mu \mathrm{g} / \mathrm{ml}$ (Tzouvelekis et al., 2012; Daikos et al., 2014) whereas other studies assign that breakpoint at $\leq 4 \mu \mathrm{g} / \mathrm{mL}$ (Miyakis et al., 2011; Tängdén, 2014). Finally, combination therapy increases the cost of treatment (Kmeid et al., 2013) and exposes the bacteria to several antimicrobials that it might develop resistance to. 
As such, the utility of combination therapy remains off-label and largely biased. Therefore, an alternative treatment approach to carbapenem-resistant bacterial infections could be the genetically guided use of $\beta$-lactam/ $\beta$-lactamase inhibitors ( $\beta$ LIs), namely, carbapenems/ $\beta$ LIs combinations.

Three $\beta$ LIs were selectively chosen to target specific mechanisms of carbapenem resistance: Avibactam against OXA-type carbapenemases, Relebactam against KPC, and calcium-EDTA against NDM. First, Avibactam is a non- $\beta$-lactambased $\beta$ LI that reversibly inactivates serine carbapenemases through the covalent acylation of the $\beta$-lactamase followed by a slow deacylation step that restores the inhibitor's core chemical structure (Ehmann et al., 2012). Avibactam is United States FDA-approved in combination with Ceftazidime (U.S. Food and Drug Administration [FDA], 2015) and is marketed as a treatment option against hospital-acquired and ventilator-associated bacterial pneumonias, and complicated intra-abdominal and urinary tract infections (Allergan, 2018). Secondly, Relebactam is also a non- $\beta$-lactam-based $\beta$ LI that targets serine carbapenemases (Hirsch et al., 2012); however, it is combined with Imipenem/Cilastatin to target Imipenem-resistant bacteria (Livermore et al., 2013; Lapuebla et al., 2015; Lob et al., 2017; Karlowsky et al., 2018). Finally, calcium disodium EDTA is a divalent metal-chelating agent that is United States FDA-approved to treat acute and chronic lead poisoning (U.S. Food and Drug Administration [FDA], 2009); however, it has been described in the literature to have the capacity to chelate the divalent cations found in the active sites of metallo- $\beta$-lactamases and has shown in vivo efficacy against Pseudomonas aeruginosa and Escherichia coli when combined with $\beta$-lactams (Aoki et al., 2010; Yoshizumi et al., 2013).

In this study, we first aim to assess the in vitro and in vivo efficacies of carbapenems in combination with the $\beta$ LIs Avibactam, Relebactam, and calcium-EDTA when targeting OXA-type carbapenemases, KPC-2, and NDM-1, respectively, and then investigate the molecular response of those genes of resistance against the carbapenem/ $\beta$ LI combinations.

\section{MATERIALS AND METHODS}

\section{Isolate Collection}

The Department of Pathology and Laboratory Medicine at the American University of Beirut Medical Center (AUBMC), Beirut, Lebanon provided all of the clinical bacterial isolates included in this study with the exception of one Salmonella spp. isolate that was provided by the Centers for Disease Control and Prevention (CDC), Atlanta, GA, United States. A total of 14 isolates were used, including: six A. baumannii, five K. pneumoniae, two E. coli, and one Salmonella spp. isolates. In addition to those isolates, three samples of presumptive carbapenem-resistant Pseudomonas aeruginosa were provided. All isolates were collected as part of routine medical sampling; thus, did not require Institutional Review Board (IRB) approval nor patient consent. The labeling of each isolate can be found in Table 1.

\section{Detection of Carbapenem Resistance Genes}

Total genomic DNA of each of the collected isolates was extracted from an overnight culture using the QIAamp ${ }^{\circledR}$ DNA Mini Kit (QIAGEN, Germany) according to manufacturer's instructions. Polymerase chain reaction (PCR) using TopTaq ${ }^{\mathrm{TM}}$ DNA Polymerase (QIAGEN, Germany) was then utilized to amplify and detect several $\beta$-lactamase-encoding genes that are implicated in carbapenem resistance. For the Enterobacteriaceae isolates, $b l a_{O X A-48}, b l a_{N D M-1}$, and $b l a_{K P C-2}$ genes were tested. For the A. baumannii isolates, bla $a_{N D M-1}$, bla OXA-23-like, bla $a_{O X-24 / 40}, b_{\text {OXA-51-like, }}$ bla $a_{O X A-58}$, and bla OXA-143-like genes were tested. For the $P$. aeruginosa isolates, $b l a_{N D M-1}$ was tested. The list of PCR primer sequences along with their target genes amplicon sizes are available in Table 2.

\section{Determination of Minimal Inhibitory Concentrations}

For each of the Enterobacteriaceae and A. baumannii isolates included in this study, the minimal inhibitory concentrations (MICs) of Imipenem (as Imipenem/Cilastatin, Tienam ${ }^{\circledR}$, Merck \& Co., Inc., Whitehouse Station, NJ, United States), Ertapenem (Invanz ${ }^{\circledR}$, Merck \& Co., Inc., Whitehouse Station, NJ, United States), and Meropenem (Meronem ${ }^{\circledR}$, AstraZeneca, Wilmington, DE, United States) were determined via antimicrobial broth microdilution in accordance with the Clinical and Laboratory Standards Institute (CLSI) guidelines (Clinical and Laboratory Standards Institute [CLSI], 2012). Escherichia coli (ATCC ${ }^{\circledR} 25922^{\mathrm{TM}}$ ) was used as a quality control strain (Clinical and Laboratory Standards Institute [CLSI], 2018c).

\section{Assessment of the in vitro Efficacy of the Carbapenem/ $\beta$-Lactamase Inhibitor Combinations}

Following initial MIC determination, the in vitro efficacies of the carbapenem/ $\beta$ LI combinations was assessed by adding fixed concentrations of the inhibitors to the experimental wells of a standard antimicrobial broth microdilution assay; thus, testing

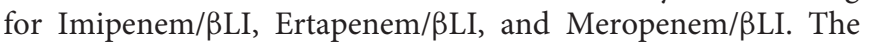
procedure followed in this assay adhered to CLSI guidelines; however, minor modifications to broth volumes were made in order to accommodate for the presence of the $\beta$ LIs while keeping the concentrations of the carbapenems and bacterial suspensions in accordance with CLSI recommendations (Clinical and Laboratory Standards Institute [CLSI], 2012).

For isolates harboring OXA-type carbapenemases, Avibactam (MedChem Express, Monmouth Junction, NJ, United States) was used as the $\beta \mathrm{LI}$ at a fixed concentration of $4 \mu \mathrm{g} / \mathrm{mL}$ (Livermore et al., 2011; Aktas et al., 2012; Sader et al., 2015). Concerning the isolates that harbored $b l a_{N D M-1}$, ethylenediaminetetraacetic acid calcium disodium salt (calcium-EDTA) (Sigma ${ }^{\circledR}$, St. Louis, MO, United States) was used as the $\beta$ LI at a fixed concentration of $32 \mu \mathrm{g} / \mathrm{mL}$ (Aoki et al., 2010; Yoshizumi et al., 2013). As for the isolate that harbored $b l a_{K P C-2}$, Relebactam (MedChem Express, 
TABLE 1 | Labels of each isolate included in the study along with their detected genes of carbapenem resistance.

\begin{tabular}{|c|c|c|c|c|c|c|c|c|c|}
\hline \multirow[t]{2}{*}{ Bacterial species } & \multirow{2}{*}{$\begin{array}{l}\text { Isolate } \\
\text { label }\end{array}$} & \multicolumn{8}{|c|}{ Genes of carbapenem resistance } \\
\hline & & blaoxA-48 & $b^{\prime} a_{N D M-1}$ & bla $_{K P C-2}$ & blaoxA-23-like & blaoxA-24/40 & blaoxA-51-like & bla $0 \times A-58$ & blaoxA-143-like \\
\hline & IMP 57 & + & - & - & & & & & \\
\hline \multirow[t]{3}{*}{ Klebsiella pneumoniae } & IMP 197 & + & - & - & & & & & \\
\hline & IMP 215 & + & - & - & & & & & \\
\hline & IMP 217 & - & + & - & & & & & \\
\hline Salmonella spp. & KPC & - & - & + & & & & & \\
\hline \multirow[t]{3}{*}{ Acinetobacter baumannii } & ACN 2090 & & - & & + & - & + & + & - \\
\hline & ACN 2209 & & - & & + & - & + & - & - \\
\hline & ACN 2273 & & - & & + & - & + & - & - \\
\hline \multirow[t]{3}{*}{ Pseudomonas aeruginosa } & PSA 41 & & - & & & & & & \\
\hline & PSA 44 & & - & & & & & & \\
\hline & PSA 45 & & - & & & & & & \\
\hline
\end{tabular}

TABLE 2 | List of target genes along with their PCR primer sequences and amplicon sizes.

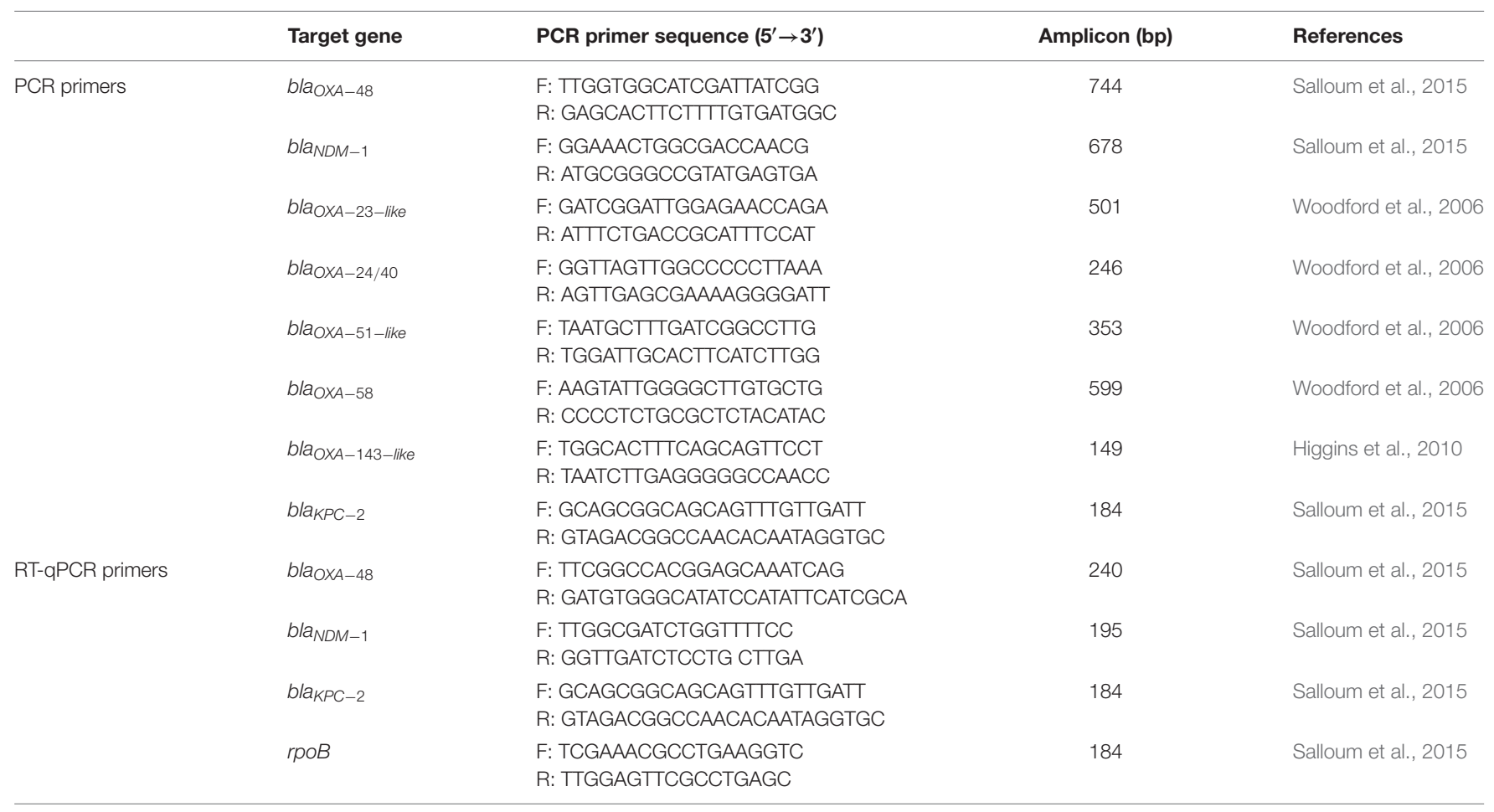

Monmouth Junction, NJ, United States) was used as the $\beta L I$ at a fixed concentration of $4 \mu \mathrm{g} / \mathrm{mL}$ (Snydman et al., 2016).

In addition, each isolate was tested against its corresponding $\beta$ LI at their aforementioned fixed concentrations without the addition of carbapenems in order to rule out any anti-bacterial activity exhibited by the inhibitors on the tested isolates.
The MICs of Imipenem (IPM), Ertapenem (ETP), and Meropenem (MEM) for all tested isolates were interpreted according to the CLSI M100 guideline (Clinical and Laboratory Standards Institute [CLSI], 2018a,b). MIC breakpoints for carbapenems in combinations with the $\beta$ LIs used in this study are currently unavailable for Enterobacteriaceae and A. baumannii. 
As such, the MIC breakpoints for Ceftazidime/Avibactam (CAZ/AVI) were used to interpret the MIC results of the carbapenem/Avibactam combinations and the MIC breakpoints for IPM, ETP, and MEM alone were used to interpret the results of their combinations with Relebactam (REL) and Ca-EDTA.

As a quality control strain, Escherichia coli (ATCC ${ }^{\circledR} 35218^{\mathrm{TM}}$ ) was used according to CLSI recommendations for $\beta$-lactam/ $\beta \mathrm{LI}$ combination testing (Clinical and Laboratory Standards Institute [CLSI], 2018c).

\section{Assessment of the in vivo Efficacy of Meropenem/ $\beta$-Lactamase Inhibitor Combinations}

The most efficacious in vitro treatment options that restored antimicrobial susceptibility of three isolates: E. coli IMP 57, K. pneumoniae IMP 216, and Salmonella spp. KPC, of which each harbored $b l a_{O X A-48}, b l a_{N D M-1}$, and $b l a_{K P C-2}$, respectively, were further investigated in animal experimentation models.

The animals involved in this study were purchased from the Animal Care Facility at the American University of Beirut. The protocols adopted in these experiments were reviewed and approved by the Institutional Animal Care and Use Committee (IACUC) at AUB under approval \#17-08-432.

A total of $150 \mathrm{BALB} / \mathrm{c}$ male mice, 6-8 weeks old, weighing 20-40 g, were used in these sets of experiments. The mice were allowed to consume food and water ad libitum throughout the experimentation period and at the end of each set of experiments, all surviving mice were humanely euthanized.

\section{Determination of the Median Lethal Dose in a BALB/c Murine Infection Model}

The procedure followed in determining the $\mathrm{LD}_{50}$ of each of the isolates E. coli IMP 57, K. pneumoniae IMP 216, and Salmonella spp. KPC involved in the animal experimentations relied on an earlier protocol (Nowotny, 1979) with an extended monitoring period. Briefly, for each of the three tested bacterial isolates, 20 mice were divided into five groups of four mice. Each group of mice were intraperitoneally injected with increasing concentrations of the tested isolate, starting with $10^{4} \mathrm{CFU}$ up to $10^{8}$ CFU. Following infection, the mice were daily monitored over a 1-week period for their survival, weight, physical appearance, and behavioral changes.

At the end of the monitoring period, the $\mathrm{LD}_{50}$ was calculated using the Spearman-Karber method (Nowotny, 1979).

\section{Investigation of Survival Rates in a BALB/c Murine Infection Model}

For each of the bacterial isolates E. coli IMP 57, K. pneumoniae IMP 216, and Salmonella spp. KPC, 30 mice were divided into five groups of six mice. The experimental setup was designed over a 7-day period (Table 3 ):

1. Group 1 acted as a positive control and received a 1-time intraperitoneal bacterial dose of $3 \times \mathrm{LD}_{50}$ suspended in tryptic soy broth (TSB) on day 1 without receiving any subsequent treatment throughout the experiment.

2. Groups 2-4 received a 1-time intraperitoneal bacterial dose of $3 \times \mathrm{LD}_{50}$ suspended in TSB on day 1 followed by intraperitoneal treatment courses once, daily, starting 1-h post-infection on day 1 and continued days 27. Treatment for Group 2 was Meropenem only, for Group 3 was Meropenem/ $\beta L I$, and for Group 4 was the corresponding $\beta$ LI only.

3. Group 5 acted as a negative control and received a 1time intraperitoneal injection of sterile TSB as a blank on day 1 without any subsequent injections throughout the experiment.

Meropenem (MEM) was used as the antimicrobial agent of choice in these sets of experiments as it was the most effective carbapenem in vitro. MEM was combined with Avibactam (AVI), calcium-EDTA (Ca-EDTA), and Relebactam (REL) against E. coli IMP 57, K. pneumoniae IMP 216 and Salmonella spp. KPC, respectively.

TABLE 3 | Mice groups and injections used in survival experimentation.

\begin{tabular}{|c|c|c|c|c|c|c|}
\hline Days & & $\begin{array}{c}\text { Group } 1 \\
\text { (P.C.) }\end{array}$ & $\begin{array}{l}\text { Group } 2 \\
\text { (MEM) }\end{array}$ & $\begin{array}{c}\text { Group } 3 \\
(\mathrm{MEM}+\beta \text { LI) }\end{array}$ & $\begin{array}{c}\text { Group } 4 \\
\text { ( } \beta \text { LI) }\end{array}$ & $\begin{array}{c}\text { Group } 5 \\
\text { (N.C.) }\end{array}$ \\
\hline \multirow[t]{8}{*}{ Day 1} & $t=0 \mathrm{hr}$ & $\begin{array}{l}\text { Bacterial } \\
\text { injection }\end{array}$ & Bacterial injection & Bacterial injection & Bacterial injection & TSB \\
\hline & $t=1 \mathrm{hr}$ & - & $\begin{array}{l}\text { Administer } \\
\text { Meropenem }\end{array}$ & $\begin{array}{c}\text { Administer } \\
\text { Meropenem + } \\
\beta \text {-lactamase } \\
\text { inhibitor }\end{array}$ & $\begin{array}{c}\text { Administer } \\
\beta \text {-lactamase } \\
\text { inhibitor }\end{array}$ & - \\
\hline & Day 2 & & & & & \\
\hline & Day 3 & & & & & \\
\hline & Day 4 & & & & & \\
\hline & Day 5 & & & & & \\
\hline & Day 6 & & & & & \\
\hline & Day 7 & & & & & \\
\hline
\end{tabular}

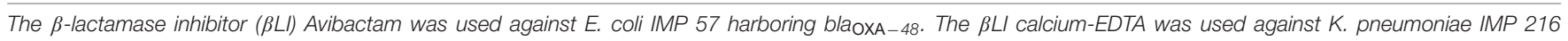

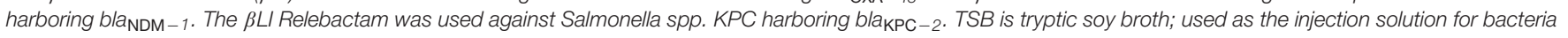
in Groups 1-4 and as a sterile blank in Group 5. 
The required dose of MEM against the tested isolates was determined according to an earlier protocol (Rahal et al., 2011b) and was $1.6 \mathrm{mg} / \mathrm{kg}$ for E. coli IMP 57, $1.75 \mathrm{mg} / \mathrm{kg}$ for Salmonella spp. KPC, and $0.115 \mathrm{mg} / \mathrm{kg}$ for K. pneumoniae IMP 216.

As for the doses of the $\beta$ LIs, AVI was administered at a 1:4 ratio with the antimicrobial agents (Endimiani et al., 2011; Levasseur et al., 2014), while Ca-EDTA and REL were each administered at an 8:1 ratio with the antimicrobial agents (Yoshizumi et al., 2013; Powles et al., 2018).

All mice were daily monitored for their survival, weight, physical appearance, and behavioral changes. Test subjects that expired prior to the end of the monitoring period had their blood cultured and API ${ }^{\circledR}$ 20E (bioMérieux, Marcy l'Etoile, France) performed on the colonies retrieved in order to confirm that the cause of death was the administered agent (Salloum et al., 2015).

\section{Assessment of the Molecular Response to the Carbapenem/ $\beta$-Lactamase Inhibitor Combinations}

Reverse transcription real-time polymerase chain reaction (RT-qPCR) was used to quantitate the expression levels of bla $a_{O X A-48}, b l a_{N D M-1}$, and $b l a_{K P C-2}$ in the tested isolates. The relative normalized expressions of the target genes were calculated using the Livak $2^{-\Delta \Delta C T}$ method (Schmittgen and Livak, 2008).

\section{Under in vitro Conditions}

Bacterial suspensions of E. coli IMP 57, K. pneumoniae IMP 216, and Salmonella spp. KPC were collected for RT-qPCR following their incubations with Meropenem alone as well as in combination with their corresponding $\beta$ LIs at MICs. An untreated sample of each bacterial isolates was used as a positive control and the $r p o B$ gene was used as a reference housekeeping gene (Salloum et al., 2015).

\section{Under in vivo Conditions}

For each of E. coli IMP 57, K. pneumoniae IMP 216, and Salmonella spp. KPC, $15 \mathrm{BALB} / \mathrm{c}$ male mice were divided into five groups of three mice and followed the same IACUCapproved infection and treatment protocols used in the survival studies above. However, mice from Groups 1-3 were then scarified via cardiac puncture under general anesthesia $4 \mathrm{~h}$ posttreatment, and their blood was collected for RT-qPCR. All blood samples were centrifuged at $1,500 \times g$ for $30 \mathrm{~min}$ and the separated plasma was retrieved for bacterial RNA extraction (Rasheed, 2016).

For both in vitro and in vivo settings, the illustra ${ }^{\mathrm{TM}}$ RNAspin Mini Kit (GE Healthcare UK Limited, Buckinghamshire, United Kingdom) was used to extract the RNA of each of the tested isolates, the iScript ${ }^{\mathrm{TM}}$ cDNA Synthesis Kit (Bio-Rad, Hercules, CA, United States) was used to synthesize complementary DNA of the extracted RNA templates, and the iTaq ${ }^{\mathrm{TM}}$ Universal SYBR ${ }^{\circledR}$ Green Supermix (Bio-Rad, Hercules, CA, United States) was used for qPCR. All kits were utilized according to their manufacturers' instructions. The real-time PCR primer sequences along with their amplicon sizes are available in Table 2.

\section{Statistical Analysis}

The logrank (Mantel-Cox) test was utilized in the survival studies analysis to calculate the statistical significance while the unpaired Student's $t$-test was used in the quantitative PCR analysis, in which $p$-values $\leq 0.05$ were considered statistically significant.

\section{RESULTS}

\section{Detection of Carbapenem Resistance Genes}

Following PCR amplification, it was observed that all collected isolates harbored at least 1 of the carbapenem resistance genes they were tested for. Consequently, bla OXA-48 was detected in five of the eight Enterobacteriaceae isolates (62.5\%), bla $a_{N D M-1}$ was detected in $2(25 \%)$, while $b a_{K P C-2}$ was only detected in one Enterobacteriaceae isolate (12.5\%). As for blaOXA-23-like and

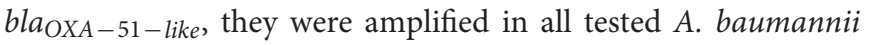
isolates (100\%), while each of blaOXA-58 and blaOXA-143-like were amplified in two of the six A. baumannii isolates (33.3\%), and neither $b l a_{O X A-24 / 40}$ nor $b l a_{N D M-1}$ were detected in any of the tested A. baumannii. Concerning bla $a_{N D M-1}$ in P. aeruginosa, none of the tested isolates harbored the gene of resistance; thus, the $P$. aeruginosa isolates were not considered further in experimentation. A summary of the identified genes is available in Table 1.

\section{Efficacy of the Carbapenem/ $\beta$-Lactamase Inhibitor Combinations in vitro}

Among the isolates that harbor bla OXA-48, IPM/AVI and MEM/AVI managed to restore carbapenem susceptibility to $100 \%$ of them while ETP/AVI restored carbapenem susceptibility to $60 \%$ of them. Similarly, among the isolates that harbor $b l a_{N D M-1}$, only testing with IPM/Ca-EDTA and MEM/Ca-EDTA resulted in $100 \%$ susceptibility, while for the isolate that harbored $b l a_{K P C-2}$, combining any of the carbapenems with REL restored carbapenem susceptibly. On the other hand, the combinations of any of the carbapenems with AVI were unsuccessful at restoring carbapenem susceptibility among the A. baumannii isolates that mainly harbored bla $a_{O X}-23$-like and bla $a_{O X}-51-l i k e$; however, they did manage to lower their MIC values by twofold in the case of ETP/AVI and at least eightfold in the cases of IPM/AVI and MEM/AVI. Minimal inhibitory concentration results of all isolates are available in Table $\mathbf{4}$ and Figure $\mathbf{1 .}$

Finally, none of the inhibitors used in this study were solely successful at inhibiting the growth of any isolate; thus, confirming that they do not exhibit antibacterial activities themselves.

\section{Efficacy of Meropenem/ $\beta$-Lactamase Inhibitor Combinations in vivo}

The median lethal dose of the tested isolates was determined as follows: $1.78 \times 10^{8} \mathrm{CFU}$ for E. coli IMP 57, $3.16 \times 10^{7} \mathrm{CFU}$ for K. pneumoniae IMP 216, and $3.16 \times 10^{8} \mathrm{CFU}$ for Salmonella spp. KPC. Recorded average mice weights are available in Supplementary Figure S1. 
TABLE 4 | MICs of Imipenem (IPM), Ertapenem (ETP), and Meropenem (MEM) with and without the $\beta$-lactamase inhibitors ( $\beta$ LI) against the tested isolates.

\begin{tabular}{|c|c|c|c|c|c|c|c|}
\hline \multirow[t]{2}{*}{ Gene of resistance } & \multirow[t]{2}{*}{ Isolate ID } & \multicolumn{6}{|c|}{$\operatorname{MIC}(\mu \mathrm{g} / \mathrm{mL})$} \\
\hline & & IPM & IPM + $\beta$ LI & ETP & $\mathrm{ETP}+\beta \mathrm{LI}$ & MEM & MEM + $\beta$ LI \\
\hline \multirow[t]{5}{*}{ blaoxA-48 } & IMP 53 & 32 & 0.25 & 128 & 2 & 16 & 0.03125 \\
\hline & IMP 57 & 64 & 1 & $>256$ & 16 & 64 & 0.25 \\
\hline & IMP 197 & $>256$ & 1 & $>256$ & 16 & 128 & 1 \\
\hline & IMP 215 & 128 & 0.5 & $>256$ & 8 & 128 & 0.125 \\
\hline & IMP 223 & 16 & 0.25 & 16 & 0.25 & 2 & 0.03125 \\
\hline \multirow[t]{2}{*}{ bla ${ }_{N D M-1}$} & IMP 216 & $>256$ & 0.5 & $>256$ & 2 & $>256$ & 0.5 \\
\hline & IMP 217 & 8 & 1 & 1 & 1 & 1 & 0.03125 \\
\hline blakPC-2 & KPC & 4 & 1 & 4 & 0.03125 & 4 & 0.03125 \\
\hline \multirow[t]{6}{*}{ blaoxA-23-like, blaoxA-51-like } & ACN 2090 & $>256$ & 32 & $>256$ & $>256$ & 128 & 32 \\
\hline & ACN 2209 & $>256$ & 32 & $>256$ & $>256$ & 128 & 32 \\
\hline & ACN 2273 & $>256$ & 8 & $>256$ & $>256$ & 128 & 8 \\
\hline & ACN 2285 & $>256$ & 32 & $>256$ & $>256$ & 128 & 32 \\
\hline & ACN 2493 & $>256$ & 32 & $>256$ & $>256$ & 128 & 32 \\
\hline & ACN 3630 & $>256$ & 8 & $>256$ & $>256$ & 128 & 8 \\
\hline
\end{tabular}

The $\beta L I$ for blaOXA-48, blaOXA-23-like, and blaOXA-51-like is Avibactam. The $\beta L I$ for blaNDM-1 is Ca-EDTA. The $\beta L I$ for blakPC-2 is Relebactam.

Concerning the survival rate of the $\mathrm{BALB} / \mathrm{c}$ mice upon infection with the tested isolates and treatment with Meropenem monotherapy in comparison to Meropenem/ $\beta$ LI combinations, the group receiving Meropenem/Avibactam against E. coli IMP 57 experienced a $100 \%$ survival rate $(p<0.0001)$ when compared to their positive control group (16.7\% survival) as well as the group receiving Meropenem monotherapy (0\% survival) and the group receiving Avibactam alone (0\% survival) (Figure 2). Similarly, the group treated with Meropenem/Relebactam against Salmonella spp. KPC experienced a 100\% survival rate $(p<0.0001)$ in comparison to their positive control group $(0 \%$ survival) in addition to the group treated with Meropenem monotherapy ( $0 \%$ survival) and the group treated with Relebactam alone ( $0 \%$ survival) (Figure 2). However, the group receiving Meropenem/calcium-EDTA against K. pneumoniae IMP 216 experienced a 16.7\% survival rate $(p=0.0009)$, identical to that of the group receiving Meropenem monotherapy, but higher than the positive control ( $0 \%$ survival) as well as the group receiving calcium-EDTA alone $(0 \%$ survival) (Figure 2).

The average weights of the different mice groups against each of the tested bacterial isolates during the survival studies are available in Supplementary Figure S2.

\section{The Molecular Response to Meropenem/ $\beta$-Lactamase Inhibitor Combinations \\ bla OXA-48}

Quantifying the in vitro relative normalized expression levels of bla $a_{O X A-48}$ in E. coli IMP 57 following the addition of Meropenem only and Meropenem/Avibactam in comparison to the positive control indicated a sixfold increase $(p=0.0024)$ in bla $a_{O X-48}$ expression when Meropenem was added, and a 10 -fold increase $(p=0.00072)$ when Meropenem/Avibactam were added. Moreover, there was a statistically significant difference $(p=0.028)$ in expression levels when comparing Meropenem to Meropenem/Avibactam (Figure 3).

Concerning the in vivo relative normalized expression levels of bla OXA-48 in E. coli IMP 57, the treatment with Meropenem only and Meropenem/Avibactam in comparison to the positive control indicated a threefold increase $(p=0.0292)$ in bla $a_{\mathrm{OX}-48}$ expression when Meropenem was administered, and a fourfold increase $(p=0.0361)$ when Meropenem/Avibactam were administered (Figure 3).

\section{bla $K P C-2$}

Measuring the in vitro relative normalized expression levels of bla $a_{K P C-2}$ in Salmonella spp. KPC following the addition of Meropenem only and Meropenem/Relebactam, when compared to the positive control, indicated a fivefold increase $(p=0.008)$ in bla $a_{K P C-2}$ expression when Meropenem was added, and a fourfold increase $(p=0.05)$ when Meropenem/Relebactam were added (Figure 3 ).

With respect to the in vivo relative normalized expression levels of bla $\mathrm{KPC}_{-2}$ in Salmonella spp. KPC, the treatment with Meropenem only and Meropenem/Relebactam, when compared to the positive control, indicated a twofold increase in $b l a_{K P C-2}$ expression when either Meropenem alone $(p=0.43)$ or Meropenem/Relebactam $(p=0.29)$ were administered (Figure 3).

\section{bla $_{N D M-1}$}

Quantifying the in vitro relative normalized expression levels of bla $a_{N D M-1}$ in K. pneumoniae IMP 216 following the addition of Meropenem only and Meropenem/calcium-EDTA when compared to the positive control indicated a fourfold increase $(p=0.134)$ in $b l a_{N D M-1}$ expression when Meropenem was added, but a significant eightfold decrease $(p=0.029)$ when Meropenem/calcium-EDTA were added. Moreover, 


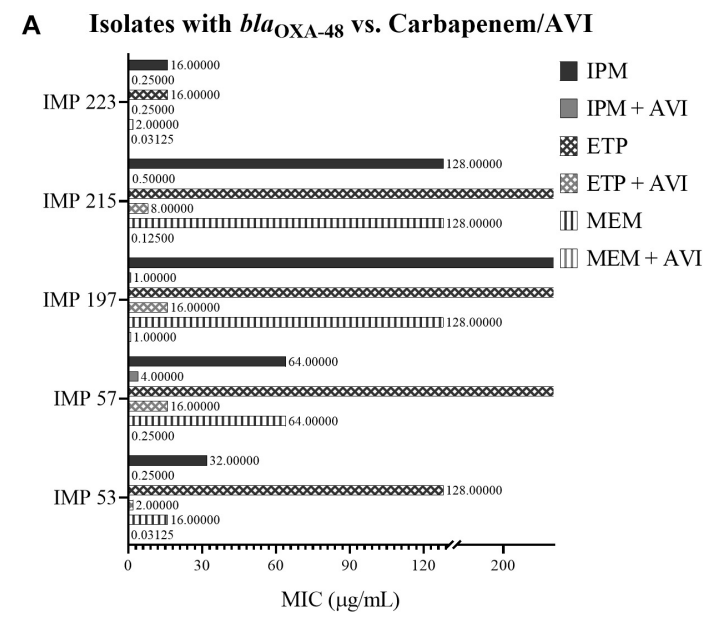

B

Isolates with bla $_{\mathrm{NDM}-1}$ vs. Carbapenem/Ca-EDTA

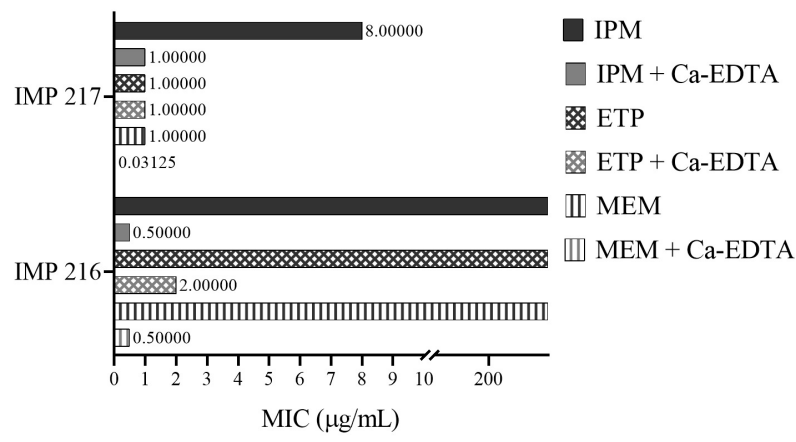

C

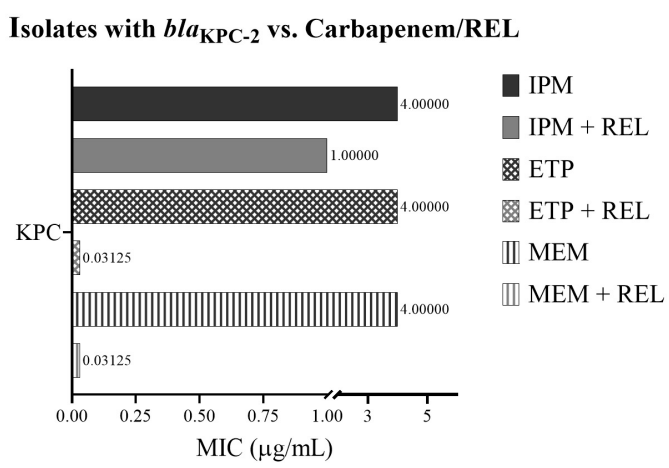

D

Isolates with bla $_{\text {OXA-23-like }} \&$ bla $_{\text {OXA-51-like }}$ vs. Carbapenem/AVI

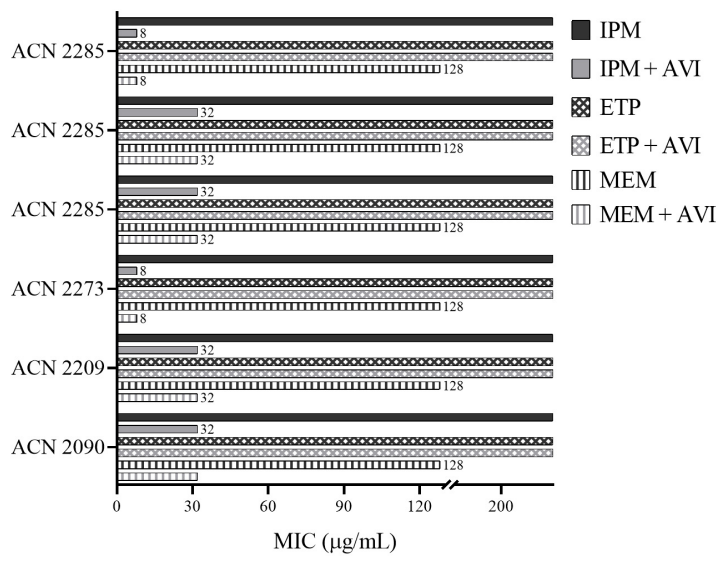

FIGURE 1 | (A) MIC of carbapenems with and without Avibactam against Enterobacteriaceae isolates that harbor blaOXA-48. (B) MIC of carbapenems with and without Ca-EDTA against Enterobacteriaceae isolates that harbor bla $\mathrm{NDM}_{-1}$. (C) MIC of carbapenems with and without Relebactam against a Salmonella spp. isolate that harbors blaKPC-2. (D) MIC of carbapenems with and without Avibactam against A. baumannii isolates that mainly harbor blaOXA-23-like and blaOXA-51-like.

\section{A}

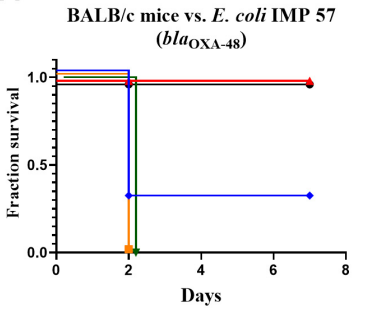

B

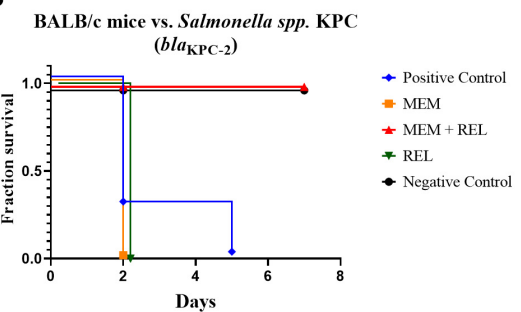

C

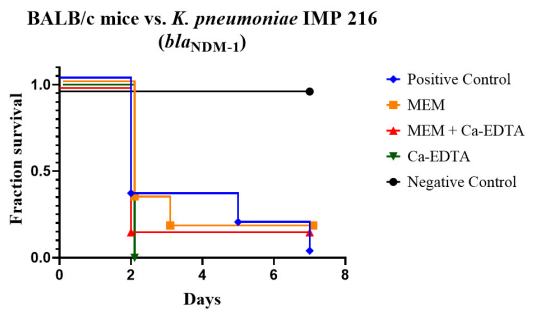

FIGURE 2 | (A) Kaplan-Meier plot showing the survival rates of treated and untreated BALB/c mice infected with E. coli IMP 57 . (B) Kaplan-Meier plot showing the survival rates of treated and untreated BALB/c mice infected with Salmonella spp. KPC. (C) Kaplan-Meier plot showing the survival rates of treated and untreated BALB/c mice infected with $K$. pneumoniae IMP 216.

there was a statistically significant difference $(p=0.021)$ in expression levels when comparing Meropenem to Meropenem/ calcium-EDTA (Figure 3).

Quantifying the in vivo relative normalized expression levels of bla $a_{N D-1}$ in K. pneumoniae IMP 216 following the treatment with Meropenem only and Meropenem/calciumEDTA when compared to the positive control indicated 2.3-fold and 3-fold decreases in bla $a_{N D M-1}$ expression when Meropenem and Meropenem/Ca-EDTA were administered, respectively (Figure 3). 


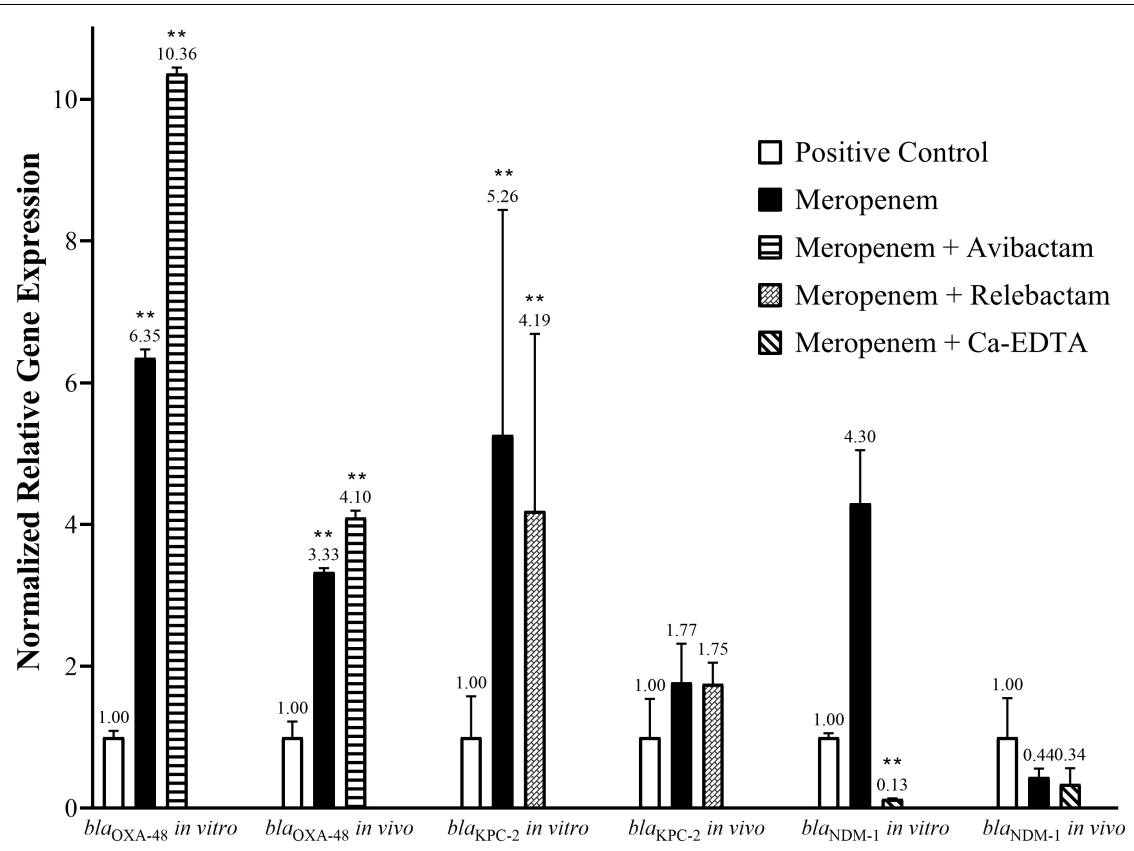

(**) Denotes statistical significance $(\mathrm{p} \leq 0.05)$

FIGURE 3 | The in vitro and in vivo normalized relative gene expression levels of blaoXA-48 in E. coli IMP 57, blakPC-2 in Salmonella spp. KPC, and bla NDM-1 in K. pneumoniae IMP 216 following the addition of Meropenem alone or in combination with Avibactam, Relebactam, and calcium-EDTA, respectively.

\section{DISCUSSION}

Antimicrobial resistance is a public health threat with major repercussions. Bacteria can rapidly develop resistance to new antimicrobial agents a few years after they become available for commercial use (Centers for Disease Control and Prevention [CDC], 2013). Carbapenem-resistant Enterobacteriaceae (CRE) and multidrug resistant Acinetobacter baumannii (MDR-A. baumannii) rank among the highest priority pathogens for research and drug discovery according to the (World Health Organization [WHO], 2017). Similarly, CREs are classified as an urgent health hazard, while MDR-A. baumannii is classified as a serious health hazard according to the (Centers for Disease Control and Prevention [CDC], 2013). Evidently, providing new and alternative solutions to treat carbapenem-resistant bacterial infections is a critical need. Although combination therapy has proven to be useful, its benefits over monotherapy remain debatable. Therefore, carbapenems/ $\beta$ LI combinations were chosen as potential alternative therapeutic solutions.

\section{Evaluating Avibactam}

When assessing the in vitro capacity of carbapenem/Avibactam combinations against CREs and MDR-A. baumannii, the addition of the $\beta \mathrm{LI}$ to carbapenems successfully restored most of the tested isolates' susceptibility to that class of antimicrobial agents.

The majority of the literature reports the combination of Ceftazidime/Avibactam against antimicrobial-resistant isolates; however, Aktas et al. (2012) have found that Imipenem/Avibactam managed to restore the susceptibility of 26 Enterobacteriaceae isolates with OXA-48. The findings reported in this study coincide with Aktas et al. (2012) regarding Imipenem/Avibactam; however, it was observed that the addition of Meropenem/Avibactam displayed considerably lower MIC values than the former combination (Table 4); thus, highlighting Meropenem/Avibactam as the more efficacious carbapenem/ $\beta \mathrm{LI}$ combination against the tested bla $\mathrm{OXA-48^{- }}$ positive Enterobacteriaceae isolates. On the other hand, Ertapenem was not as effective as Imipenem or Meropenem when combined with Avibactam against OXA-48 as it only managed to restore the susceptibility of three Enterobacteriaceae isolates. Finally, none of the carbapenem/Avibactam combinations used in this study managed to restore the susceptibility of any of the A. baumannii isolates that mainly harbored bla $a_{O X A-23-l i k e}$ and bla $a_{O X A-51-l i k e}$ (Table 1), which is consistent with the reported literature (Thaden et al., 2016).

Concerning the in vivo experiments, assessing the survival rate of murine infection models against $E$. coli IMP 57 that harbors bla OXA $_{-48}$ and attempting to treat the animals with Meropenem/Avibactam has not been documented in the literature yet. As such, experimental design and dosage determinations were guided by earlier studies with similar target parameters (Rahal et al., 2011a; Levasseur et al., 2014; Salloum et al., 2015). The in vitro and in vivo results observed were compatible with minimal discrepancy. The group of mice that was infected with E. coli IMP 57 and treated with Meropenem/Avibactam showed a $100 \%$ survival rate, while that of the groups that received Meropenem or Avibactam monotherapy experienced a $0 \%$ survival rate (Figure 2). These findings prove the efficacy of Meropenem/Avibactam against 
OXA-48 among Enterobacteriaceae, and that the concentration of Meropenem to Avibactam at a 4:1 ratio is effective. Concerning the positive control group that was infected with E. coli IMP 57 without receiving any treatment, the survival of one mouse might have been due to a technical error during the intraperitoneal injection of the bacterial inoculum (Steward et al., 1968).

At the molecular level, both in vitro and in vivo results showed similar trends in relative blaOXA-48 expression, signifying the consistency of Meropenem/Avibactam activity despite the differences in environments. Meropenem appears to have induced the expression of blaOXA-48; however, its statistically significant overexpression upon the addition of Meropenem/Avibactam could be due to a synergistic relationship between Meropenem and Avibactam since it has been previously proven that Avibactam does not induce the production of $\beta$-lactamases by itself (Miossec et al., 2013), especially at concentrations below $32 \mu \mathrm{g} / \mathrm{mL}$ (Livermore et al., 2017); thus, had there not been synergism between them, the level of bla OXA-48 expression upon the addition of Meropenem/Avibactam would be similar to that following the addition of Meropenem alone. Additional data supporting the possibility of having synergism between Avibactam and Meropenem is their high affinity to the same penicillin-binding protein 2 (PBP2) in E. coli (Davies et al., 2008; Asli et al., 2016). It is worthy to note that regardless of the overexpressed carbapenemase, a low concentration of Avibactam was sufficient to inhibit the enzyme and permit the activity of Meropenem. It is possible that such a low concentration of Avibactam was sufficient due to the reversible inhibition property that it displays (Ehmann et al., 2012).

\section{Evaluating Relebactam}

The in vitro testing of REL in combination with carbapenems has demonstrated its effectiveness against the tested Salmonella spp. KPC isolate as its susceptibility to IPM, ETP, and MEM was restored. These findings are in line with a previous study that reported IPM/REL as an effective combination against $78.5 \%$ Imipenem-non-susceptible non-Proteeae Enterobacteriaceae (Karlowsky et al., 2018). Furthermore, carbapenems/Relebactam showed targeted potency against KPC-2 and that also complements an earlier study that reported Imipenem/Relebactam restoring the susceptibility of $97 \%$ of KPC-producing K. pneumoniae isolates (Lapuebla et al., 2015). In addition, the safety and efficacy of Imipenem/Cilastatin/Relebactam versus Imipenem/Cilastatin alone have been tested in clinical trials among patients with complicated intra-abdominal infections (cIAI) (Lucasti et al., 2016) and complicated urinary tract infections (cUTI) (Sims et al., 2017). Both of those trials concluded that treating patients suffering from cIAI or cUTI using IPM/REL resulted in high rates of favorable microbiological and clinical responses at the end of treatment although IPM/REL was non-inferior to IPM alone. Despite the majority of the literature reporting Imipenem/Relebactam combinations, the results presented here provide Ertapenem and Meropenem as more efficacious alternatives as they displayed lower MICs, reaching 0.03125 $\mu \mathrm{g} / \mathrm{mL}$, upon their combination with Relebactam (Table 4).
With regards to the in vivo murine survival studies, the $100 \%$ survival rate of the mice treated with Meropenem/Relebactam against the $b a_{\mathrm{KPC}-2}$-positive bacterial isolate in comparison to the $0 \%$ survival rates of their control groups matched the in vitro results and mimicked those of Meropenem/Avibactam against bla $a_{O X A-48}$ (Figure 2), as this has also not yet been documented in the literature. These findings support the potency of the Meropenem/Relebactam combination against the tested Salmonella spp. KPC isolate in vivo as well as the efficacy of the 8:1 dosing ratio of Relebactam to Meropenem and the route of antimicrobial administration.

At the molecular level, Meropenem seemed to have induced the overexpression of bla $a_{K C-2}$ in vitro regardless of the potentiating effect of Relebactam, as the latter is not a $\beta$-lactamase inducer by itself (Livermore et al., 2017); however, the insignificant decrease in gene expression levels due to the addition of Meropenem/Relebactam as compared to Meropenem alone could be due to the inhibitory effect that Relebactam exerted on KPC-2 without it having a compensatory mechanism that is similar to the one observed in bla OXA-48. On the other hand, the near-identical expression levels of $b l a_{K P C-2}$ in vivo upon the treatment with Meropenem monotherapy in comparison to Meropenem/Relebactam does not correlate with its in vitro gene expression observations despite displaying potency in the murine infection model. That discrepancy might be due to the change of environment between in vitro and in vivo settings, which could have altered the behavior of $b l a_{K P C-2}$ in response to treatment; however, that conclusion requires further investigation.

\section{Evaluating Calcium-EDTA}

The in vitro assessment of Ca-EDTA in combination with carbapenems displayed high susceptibility rates amongst the tested isolates that harbor $b a_{N D M-1}$ (Table 4). These results validate an earlier study that investigated the efficacy of Imipenem and Meropenem in combination with Ca-EDTA against NDM-1-positive $K$. pneumoniae and E. coli isolates (Yoshizumi et al., 2013); however, in contrast to that study, the Imipenem and Meropenem combinations with Ca-EDTA successfully lowered MIC values by a maximum of 512 -fold, to reach $0.03125 \mu \mathrm{g} / \mathrm{mL}$ for Meropenem/Ca-EDTA, whereas those in Yoshizumi et al. (2013) were lowered by 256 -fold at most, reaching $1 \mu \mathrm{g} / \mathrm{mL}$. Finally, the combination of Ca-EDTA with Ertapenem was not as successful as with the other two carbapenems since it failed to render any of the tested isolates susceptible although it did lower the MIC of one isolate by at least 128 -fold. These finding highlight Meropenem/Ca-EDTA as the more efficacious combination against the tested isolates.

Concerning the in vivo murine survival studies, Meropenem/Ca-EDTA did not demonstrate any added efficacy when compared to Meropenem monotherapy as the mice from both groups achieved identical survival rates of $16.7 \%$ whereas the positive control and Ca-EDTA monotherapy groups each resulted in $0 \%$ survival. A similar acute lethal septicemia experiment involving an NDM-1-positive E. coli isolate was performed by Yoshizumi et al. (2013); however, bacterial burden was assessed in that study and it was concluded that 
Imipenem/Ca-EDTA significantly reduced the bacterial burden in the blood and liver of neutropenic mice. In this study, the Meropenem/Ca-EDTA combination might have failed to demonstrate additional potency due to a potentially insufficient dose or an inappropriate intraperitoneal route of administration as Ca-EDTA might have chelated non-specific divalent cations found in the mice's bodies.

With regards to the gene expression levels, the addition of Meropenem in vitro did not seem to significantly alter the expression of bla $a_{N D M-1}$; however, supplementing Ca-EDTA to Meropenem managed to significantly suppress the expression of the gene by approximately eightfold. These findings directly explain the observed decrease in MIC levels as Meropenem/Ca-EDTA successfully inhibited the carbapenemase and restored the isolate's susceptibility. On the other hand, the use of Meropenem/Ca-EDTA as a treatment option in the murine infection model did not cause a significant difference in the expression levels of $b l a_{N D M-1}$ as compared to the positive control, but instead resulted in a gene expression level that is similar to the one due to Meropenem monotherapy. This latter observation supports the inefficacy of Meropenem/Ca-EDTA that was observed in the survival studies, as Ca-EDTA does not appear to have exerted its inhibitory effect on NDM-1 in vivo, leaving Meropenem to act on its own, which resulted in similar gene expression levels under both treatment conditions.

\section{CONCLUSION}

Utilizing carbapenems, namely Meropenem, with the novel $\beta$-lactamase inhibitors Avibactam, Relebactam, and Ca-EDTA has proven to be capable of restoring carbapenem susceptibility among bacterial isolates that express the highly clinically relevant carbapenemases OXA-48, KPC-2, and NDM-1. Taken together, the in vitro, in vivo, and gene expression data encourage further investigating Meropenem/Avibactam and Meropenem/Relebactam as potential targeted therapeutic options against OXA-48 and KPC, respectively. However, a multi-faceted approach with a larger sample size and greater genetic diversity is required for both phenotypic and genotypic testing before proceeding into further preclinical and clinical development.

\section{DATA AVAILABILITY}

All datasets generated for this study are included in the manuscript and/or the Supplementary Files.

\section{REFERENCES}

Aktas, Z., Kayacan, C., and Oncul, O. (2012). In vitro activity of avibactam (nxl104) in combination with beta-lactams against gram-negative bacteria, including oxa-48 beta-lactamase-producing Klebsiella pneumoniae. Int. J. Antimicrob. Agents 39, 86-89. doi: 10.1016/j.ijantimicag.2011. 09.012

Allergan. (2018). Avycaz Indications and Usage. Irvine, CA: Allergan Inc.

\section{ETHICS STATEMENT}

This study was carried out in accordance with the recommendations of the Institutional Animal Care and Use Committee at the American University of Beirut. The protocol was approved by the Institutional Animal Care and Use Committee at the American University of Beirut under approval \#17-08-432.

\section{AUTHOR CONTRIBUTIONS}

$\mathrm{BH}$ designed and executed the experiments, analyzed the data, and wrote and edited the manuscript. SR designed the experiments, analyzed the data, and reviewed and edited the manuscript. AAF provided scientific feedback, analyzed the data, and reviewed and edited the manuscript. GA performed phenotypic bacteriological testing, supplied the bacterial isolates, provided scientific feedback, and reviewed and edited the manuscript. GM conceived the study, designed the experiments, analyzed the data, and edited the overall manuscript.

\section{FUNDING}

This study was funded by the Medical Practice Plan (MPP) at the Faculty of Medicine of the American University of Beirut. All interpretations, conclusions, and recommendations resulting from this study are those of the authors and are not necessarily endorsed by the funding body.

\section{SUPPLEMENTARY MATERIAL}

The Supplementary Material for this article can be found online at: https://www.frontiersin.org/articles/10.3389/fmicb. 2019.00933/full\#supplementary-material

FIGURE S1 | (A) Recorded average mice weights in their different groups during $\mathrm{LD}_{50}$ determination of $E$. coli IMP 57. (B) Recorded average mice weights in their different groups during $L D_{50}$ determination of $K$. pneumoniae IMP 216. (C) Recorded average mice weights in their different groups during $L D_{50}$ determination of Salmonella spp. KPC.

FIGURE S2 | (A) Recorded average mice weights in their different groups during survival studies against E. coli IMP 57. (B) Recorded average mice weights in their different groups during survival studies against $K$. pneumoniae IMP 216. (C) Recorded average mice weights in their different groups during survival studies against Salmonella spp. KPC.

Aoki, N., Ishii, Y., Tateda, K., Saga, T., Kimura, S., Kikuchi, Y., et al. (2010). Efficacy of calcium-EDTA as an inhibitor for metallo$\beta$-lactamase in a mouse model of Pseudomonas aeruginosa pneumonia. Antimicrob. Agents Chemother. 54, 4582-4588. doi: 10.1128/AAC. 00511-10

Araj, G. F., and Zaatari, G. S. (2015). Antimicrobial Susceptibility Profiles of Bacterial Isolates at the American University of Beirut Medical Center. Beirut: Aubmc. 
Araj, G. F., and Zaatari, G. S. (2018). Antimicrobial Susceptibility Profiles of Bacterial Isolates at the American University of Beirut Medical Center. Beirut: Aubmc.

Asli, A., Brouillette, E., Krause, K. M., Nichols, W. W., and Malouin, F. (2016). Distinctive binding of avibactam to penicillin-binding proteins of gram-negative and gram-positive bacteria. Antimicrob. Agents Chemother. 60, 752-756. doi: 10.1128/AAC.02102-15

Baroud, M., Dandache, I., Araj, G. F., Wakim, R., Kanj, S., Kanafani, Z., et al. (2013). Underlying mechanisms of carbapenem resistance in extendedspectrum $\beta$-lactamase-producing Klebsiella pneumoniae and Escherichia coli isolates at a tertiary care centre in Lebanon: role of OXA-48 and NDM-1 carbapenemases. Int. J. Antimicrob. Agents 41, 75-79. doi: 10.1016/j.ijantimicag. 2012.08.010

Centers for Disease Control and Prevention [CDC] (2013). Antibiotic Resistance Threats in the United States, 2013. Atlanta, GA: Centers for Disease Control and Prevention.

Chamoun, K., Farah, M., Araj, G., Daoud, Z., Moghnieh, R., Salameh, P., et al. (2016). Surveillance of antimicrobial resistance in lebanese hospitals: retrospective nationwide compiled data. Int. J. Infect. Dis. 46, 64-70. doi: 10. 1016/j.ijid.2016.03.010

Clinical, and Laboratory Standards Institute [CLSI] (2012). Methods for Dilution Antimicrobial Susceptibility Tests for Bacteria That Grow Aerobically; Approved Standard. .

Clinical, and Laboratory Standards Institute [CLSI] (2018a). Performance Standards for Antimicrobial Susceptibility Testing. .

Clinical, and Laboratory Standards Institute [CLSI] (2018b). Performance Standards for Antimicrobial Susceptibility Testing.

Clinical, and Laboratory Standards Institute [CLSI] (2018c). Performance Standards for Antimicrobial Susceptibility Testing.

Daikos, G. L., Tsaousi, S., Tzouvelekis, L. S., Anyfantis, I., Psichogiou, M., Argyropoulou, A., et al. (2014). Carbapenemase-producing Klebsiella pneumoniae bloodstream infections: lowering mortality by antibiotic combination schemes and the role of carbapenems. Antimicrob. Agents Chemother. 58, 2322-2328. doi: 10.1128/AAC.02166-13

Davies, T. A., Shang, W., Bush, K., and Flamm, R. K. (2008). Affinity of doripenem and comparators to penicillin-binding proteins in Escherichia coli and Pseudomonas aeruginosa. Antimicrob. Agents Chemother. 52, 1510-1512. doi: 10.1128/AAC.01529-07

Djahmi, N., Dunyach-Remy, C., Pantel, A., Dekhil, M., Sotto, A., and Lavigne, J.-P. (2014). Epidemiology of carbapenemase-producing Enterobacteriaceae and Acinetobacter baumannii in mediterranean countries. BioMed Res. Int. 2014:305784.

Ehmann, D. E., Jahiæ, H., Ross, P. L., Gu, R.-F., Hu, J., Kern, G., et al. (2012) Avibactam is a covalent, reversible, non- $\beta$-lactam $\beta$-lactamase inhibitor. Proc. Natl. Acad. Sci. U.S.A. 109, 11663-11668. doi: 10.1073/pnas.1205073109

Endimiani, A., Hujer, K. M., Hujer, A. M., Pulse, M. E., Weiss, W. J., and Bonomo, R. A. (2011). Evaluation of ceftazidime and NXL104 in two murine models of infection due to KPC-producing Klebsiella pneumoniae. Antimicrob. Agents Chemother. 55, 82-85. doi: 10.1128/AAC.01198-10

Higgins, P. G., Lehmann, M., and Seifert, H. (2010). Inclusion of OXA-143 primers in a multiplex polymerase chain reaction (PCR) for genes encoding prevalent OXA carbapenemases in Acinetobacter spp. Int. J. Antimicrob. Agents 35:305.

Hirsch, E. B., Ledesma, K. R., Chang, K.-T., Schwartz, M. S., Motyl, M. R., and Tam, V. H. (2012). In Vitro Activity of MK-7655, a novel $\beta$-Lactamase inhibitor, in combination with imipenem against carbapenem-resistant gram-negative bacteria. Antimicrob. Agents Chemother. 56, 3753-3757. doi: 10.1128/AAC. 05927-11

Karlowsky, J. A., Lob, S. H., Kazmierczak, K. M., Young, K., Motyl, M. R., and Sahm, D. F. (2018). In vitro activity of imipenem-relebactam against clinical isolates of gram-negative bacilli isolated in hospital laboratories in the united states as part of the smart 2016 program. Antimicrob. Agents Chemother. 62:e169-18. doi: 10.1128/AAC.00169-18

Kmeid, J. G., Youssef, M. M., Kanafani, Z. A., and Kanj, S. S. (2013). Combination therapy for gram-negative bacteria: what is the evidence? Expert Rev. Anti Infect. Ther. 11, 1355-1362. doi: 10.1586/14787210.2013.846215

Lapuebla, A., Abdallah, M., Olafisoye, O., Cortes, C., Urban, C., Landman, D., et al. (2015). Activity of imipenem with relebactam against gram-negative pathogens from New York city. Antimicrob. Agents Chemother. 59, 5029-5031. doi: 10.1128/AAC.00830-15

Levasseur, P., Girard, A.-M., Lavallade, L., Miossec, C., Pace, J., and Coleman, K. (2014). Efficacy of a ceftazidime-avibactam combination in a murine model of septicemia caused by Enterobacteriaceae species producing ampc or extendedspectrum $\beta$-lactamases. Antimicrob. Agents Chemother. 58, 6490-6495. doi: 10.1128/AAC.03579-14

Livermore, D. M., Jamrozy, D., Mushtaq, S., Nichols, W. W., Young, K., and Woodford, N. (2017). AmpC $\beta$-lactamase induction by avibactam and relebactam. J. Antimicrob. Chemother. 72, 3342-3348. doi: 10.1093/jac/dkx298

Livermore, D. M., Mushtaq, S., Warner, M., Zhang, J., Maharjan, S., Doumith, M., et al. (2011). Activities of NXL104 combinations with ceftazidime and aztreonam against carbapenemase-producing Enterobacteriaceae. Antimicrob. Agents Chemother. 55, 390-394. doi: 10.1128/AAC.00756-10

Livermore, D. M., Warner, M., and Mushtaq, S. (2013). Activity of MK7655 combined with imipenem against Enterobacteriaceae and Pseudomonas aeruginosa. J. Antimicrob. Chemother. 68, 2286-2290.

Livorsi, D. J., Chorazy, M. L., Schweizer, M. L., Balkenende, E. C., Blevins, A. E., Nair, R., et al. (2018). A systematic review of the epidemiology of carbapenemresistant Enterobacteriaceae in the united states. Antimicrob. Resist. Infect. Control 7:55.

Lob, S. H., Hackel, M. A., Kazmierczak, K. M., Young, K., Motyl, M. R., Karlowsky, J. A., et al. (2017). In vitro activity of imipenem-relebactam against gramnegative eskape pathogens isolated by clinical laboratories in the united states in 2015 (results from the smart global surveillance program). Antimicrob. Agents Chemother. 61, e2209-e2216. doi: 10.1128/AAC.02209-16

Lucasti, C., Vasile, L., Sandesc, D., Venskutonis, D., Mcleroth, P., Lala, M., et al. (2016). Phase 2, dose-ranging study of relebactam with imipenem-cilastatin in subjects with complicated intra-abdominal infection. Antimicrob. Agents Chemother. 60, 6234-6243. doi: 10.1128/AAC.00633-16

Meletis, G. (2016). Carbapenem resistance: overview of the problem and future perspectives. Ther. Adv. Infect. Dis. 3, 15-21. doi: 10.1177/2049936115621709

Miossec, C., Claudon, M., Levasseur, P., and Black, M. T. (2013). The $\beta$-lactamase inhibitor avibactam (NXL104) does not induce ampC $\beta$-lactamase in Enterobacter cloacae. Infect. Drug Resist. 6, 235-240. doi: 10.2147/IDR. S53874

Miyakis, S., Pefanis, A., and Tsakris, A. (2011). The challenges of antimicrobial drug resistance in greece. Clin. Infect. Dis. 53, 177-184.

Murray, P. R., Rosenthal, K. S., and Pfaller, M. A. (2016). Enterobacteriaceae in Medical Microbiology, 8th Edn. Philadelphia, PA: Elsevier, 251-264.

Nordmann, P., Naas, T., and Poirel, L. (2011). Global spread of carbapenemaseproducing Enterobacteriaceae. Emerg. Infect. Dis. 17, 1791-1798. doi: 10.3201/ eid1710.110655

Nowotny, A. (1979). Determination of Toxicity in Basic Exercises in Immunochemistry: A Laboratory Manual. Berlin: Springer, 303-305.

Paul, M., Carmeli, Y., Durante-Mangoni, E., Mouton, J. W., Tacconelli, E., Theuretzbacher, U., et al. (2014). Combination therapy for carbapenemresistant Gram-negative bacteria. J. Antimicrob. Chemother. 69, 2305-2309.

Powles, M. A., Galgoci, A., Misura, A., Colwell, L., Dingley, K. H., Tang, W., et al. (2018). In vivo efficacy of relebactam (MK-7655) in combination with imipenem-cilastatin in murine infection models. Antimicrob. Agents Chemother. 62:e2577-17. doi: 10.1128/AAC.02577-17

Queenan, A. M., and Bush, K. (2007). Carbapenemases: the versatile $\beta$-lactamases. Clin. Microbiol. Rev. 20, 440-458.

Queenan, A. M., Pillar, C. M., Deane, J., Sahm, D. F., Lynch, A. S., Flamm, R. K., et al. (2012). Multidrug resistance among Acinetobacter spp. in the USA and activity profile of key agents: results from CAPITAL surveillance 2010 Diagn. Microbiol. Infect. Dis. 73, 267-270. doi: 10.1016/j.diagmicrobio.2012. 04.002

Rahal, E. A., Kazzi, N., Kanbar, A., Abdelnoor, A. M., and Matar, G. M. (2011a). Role of rifampicin in limiting Escherichia coli O157:H7 Shiga-like toxin expression and enhancement of survival of infected BALB/c mice. Int. J. Antimicrob. Agents 37, 135-139. doi: 10.1016/j.ijantimicag.2010.10.009

Rahal, E. A., Kazzi, N., Sabra, A., Abdelnoor, A. M., and Matar, G. M. (2011b). Decrease in shiga toxin expression using a minimal inhibitory concentration of rifampicin followed by bactericidal gentamicin treatment enhances survival of Escherichia coli O157:H7-infected BALB/c mice. Ann. Clin. Microbiol. Antimicrob. 10:34. doi: 10.1186/1476-0711-10-34 
Rasheed, S. S. (2016). The Effect of Micafungin and Anti-Bacterial Agents on Pseudomonas Aeruginosa Biofilm Formation in Balb/C Mice. M.Sc. .

Sader, H. S., Castanheira, M., Flamm, R. K., Mendes, R. E., Farrell, D. J., and Jones, R. N. (2015). Ceftazidime/avibactam tested against Gram-negative bacteria from intensive care unit (ICU) and non-ICU patients, including those with ventilator-associated pneumonia. Int. J. Antimicrob. Agents 46, 53-59. doi: 10. 1016/j.ijantimicag.2015.02.022

Sahuquillo-Arce, J. M., Hernández-Cabezas, A., Yarad-Auad, F., Ibáñez-Martínez, E., Falomir-Salcedo, P., and Ruiz-Gaitán, A. (2015). Carbapenemases: a worldwide threat to antimicrobial therapy. World J. Pharmacol. 4, 75-95.

Salloum, N. A., Kissoyan, K. A. B., Fadlallah, S., Cheaito, K., Araj, G. F., Wakim, R., et al. (2015). Assessment of combination therapy in BALB/c mice injected with carbapenem-resistant Enterobacteriaceae strains. Front. Microbiol. 6:999. doi: $10.3389 /$ fmicb. 2015.00999

Satlin, M. J., Chen, L., Patel, G., Gomez-Simmonds, A., Weston, G., Kim, A. C., et al. (2017). Multicenter clinical and molecular epidemiological analysis of bacteremia due to carbapenem-resistant Enterobacteriaceae (CRE) in the cre epicenter of the united states. Antimicrob. Agents Chemother. 61, e2349-16.

Schmittgen, T. D., and Livak, K. J. (2008). Analyzing real-time PCR data by the comparative CT method. Nat. Protoc. 3, 1101-1108.

Sims, M., Mariyanovski, V., Mcleroth, P., Akers, W., Lee, Y.-C., Pedley, A., et al. (2017). Prospective, randomized, double-blind, Phase 2 dose-ranging study comparing efficacy and safety of imipenem/cilastatin plus relebactam with imipenem/cilastatin alone in patients with complicated urinary tract infections. J. Antimicrob. Chemother. 72, 2616-2626. doi: 10.1093/jac/dkx139

Snydman, D. R., Jacobus, N. V., and Mcdermott, L. A. (2016). In vitro evaluation of the activity of imipenem-relebactam against 451 recent clinical isolates of Bacteroides group and related Species. Antimicrob. Agents Chemother. 60, 6393-6397. doi: 10.1128/AAC.01125-16

Steward, J. P., Ornellas, E. P., Beernink, K. D., and Northway, W. H. (1968). Errors in the technique of intraperitoneal injection of mice. Appl. Microbiol. $16,1418-1419$.

Tängdén, T. (2014). Combination antibiotic therapy for multidrug-resistant gramnegative bacteria. Ups. J. Med. Sci. 119, 149-153. doi: 10.3109/03009734.2014. 899279

Thaden, J. T., Pogue, J. M., and Kaye, K. S. (2016). Role of newer and re-emerging older agents in the treatment of infections caused by carbapenem-resistant Enterobacteriaceae. Virulence 8, 403-416. doi: 10.1080/21505594.2016.1207834

The Medical Letter, I. (2013). Issue 131: drugs for bacterial infections. Treat. Guidel. Med. Lett. 11, 65-74.
Tumbarello, M., Viale, P., Viscoli, C., Trecarichi, E. M., Tumietto, F., Marchese, A., et al. (2012). Predictors of mortality in bloodstream infections caused by Klebsiella pneumoniae carbapenemase-producing K. pneumoniae: importance of combination therapy. Clin. Infect. Dis. 55, 943-950. doi: 10.1093/cid/ cis588

Tzouvelekis, L. S., Markogiannakis, A., Psichogiou, M., Tassios, P. T., and Daikos, G. L. (2012). Carbapenemases in Klebsiella pneumoniae and other Enterobacteriaceae: an evolving crisis of global dimensions. Clin. Microbiol. Rev. 25, 682-707. doi: 10.1128/CMR.05035-11

U.S. Food and Drug Administration [FDA] (2009). Calcium Disodium Versenate Edetate Calcium Disodium Injection. (Silver Spring, MD: U.S. Food and Drug Administration).

U.S. Food and Drug Administration [FDA] (2015). AVYCAZ (ceftazidimeavibactam) for Injection, for intravenous use. (Silver Spring, MD: U.S. Food and Drug Administration).

Woodford, N., Ellington, M. J., Coelho, J. M., Turton, J. F., Ward, M. E., Brown, S., et al. (2006). Multiplex PCR for genes encoding prevalent OXA carbapenemases in Acinetobacter spp. Int. J. Antimicrob. Agents 27, 351-353.

World Health Organization [WHO] (2017). Global Priority List of AntibioticResistant Bacteria to Guide Research, Discovery, and Development of New Antibiotics WHO Priority Pathogents List for R®D of New Antibiotics. Geneva: World Health Organization.

Yoshizumi, A., Ishii, Y., Livermore, D. M., Woodford, N., Kimura, S., Saga, T., et al. (2013). Efficacies of calcium-EDTA in combination with imipenem in a murine model of sepsis caused by Escherichia coli with NDM-1 beta-lactamase. J. Infect. Chemother. 19, 992-995. doi: 10.1007/s10156-012-0528-y

Yu, W.-L., and Chuang, Y.-C. (2016). Clinical Features, Diagnosis, and Treatment of Klebsiella Pneumoniae Infection. Waltham, MA: UpToDate Inc.

Conflict of Interest Statement: The authors declare that the research was conducted in the absence of any commercial or financial relationships that could be construed as a potential conflict of interest.

Copyright (c) 2019 El Hafi, Rasheed, Abou Fayad, Araj and Matar. This is an openaccess article distributed under the terms of the Creative Commons Attribution License (CC BY). The use, distribution or reproduction in other forums is permitted, provided the original author(s) and the copyright owner(s) are credited and that the original publication in this journal is cited, in accordance with accepted academic practice. No use, distribution or reproduction is permitted which does not comply with these terms. 